Pure Appl. Chem., Vol. 72, No. 10, pp. 1819-1834, 2000.

(C) 2000 IUPAC

\title{
Thermodynamics of structured fluids. Hard science for soft materials*
}

\author{
John M. Prausnitz \\ Chemical Engineering Department, University of California, Berkeley and \\ Chemical Sciences Division, Lawrence Berkeley National Laboratory, Berkeley, CA \\ 94720 USA
}

\begin{abstract}
At liquid-like densities, molecules of complex fluids can assume a variety of structures (or positions) in space; when the molecules contain many atoms as, for example, in polymers, that variety becomes very large. Further, when confined to a narrow space, it is possible to achieve structures that are not normally observed. Thanks to recent advances in statistical mechanics and molecular physics, and thanks to increasingly fast computers, it is now possible to calculate a fluid's structure, that is, the positions of molecules at equilibrium under given conditions. Calculation of fluid structure is useful because thermodynamic properties depend strongly on that structure, leading to possible applications for new materials. Three examples illustrate some recent developments; each example is presented only schematically (with a minimum of equations) to indicate the physical basis of the mathematical description. The first example considers the effect of branching on self-assembly (micellization) of copolymers (with possible long-range applications in medicine). The second and third examples consider the effect of confinement on fluid structure: first, crystallization in a narrow, confined space to produce a desired crystal structure (with possible applications for light-emitting diodes) and second, suppression of micellization of a diblock copolymer in a thin film (with possible application in lithography). Whenever possible, theoretical calculations are compared with experimental results.
\end{abstract}

\section{INTRODUCTION}

Since the pioneering work of Gibbs about 125 years ago, chemical thermodynamics has generated a vast literature, dozens of textbooks, and thousands of articles in many languages. The overwhelming majority of these publications is concerned with experimental or theoretical studies of relatively simple substances such as hydrocarbons, petrochemicals, salts, metals, and so on; historically, much less attention has been given to the thermodynamic properties of complex materials like gels, biomacromolecules, micelles, colloids, block copolymers, and similar substances that are often called soft materials. While such materials abound in nature and technology, application of chemical thermodynamics to soft materials has been delayed because of experimental difficulties and because, until recently, there were few theoretical models available for describing assemblies of complicated molecules.

To utilize these evolving theoretical models, we require statistical mechanics. About 50 years ago, the status of statistical mechanics was well-summarized by a Nobel laureate, physicist Eugene Wigner:

"With thermodynamics, we can calculate many things-roughly.

With kinetic theory, we can calculate a few things-approximately.

With statistical mechanics, we can calculate (almost) nothing-exactly".

\footnotetext{
*Plenary lecture presented at the $16^{\text {th }}$ IUPAC Conference on Chemical Thermodynamics (ICCT-2000), Halifax, Nova Scotia, Canada, 6-11 August 2000. Other presentations are published in this issue, pp. 1799-2082.
} 
That was two generations ago. Now, thanks to much progress in theoretical physical chemistry and to the ever-rising power of computers, Wigner's pessimistic comment is no longer valid. Statistical mechanics is now not only applicable to a wide variety of relatively simple substances but, in addition, we can clearly discern a new era where statistical mechanics can be used to describe the properties of those complex soft materials found in nature or synthesized by clever experimental scientists for high technology. Three examples illustrate this coming era. They are chosen to indicate some areas of current research and to encourage young chemical thermodynamicists to give attention to new problems, toward contributing to the development of the emerging science of soft materials. In choosing these examples, not from my own work but from the work of others that I admire, I resemble the rooster who showed an ostrich egg to the hen and said, "I am not complaining. I only want to show you what's possible."

\section{FORMATION OF AN ASSEMBLED STRUCTURE WITH BRANCHED (COMB) RANDOM COPOLYMERS}

It is common knowledge that amphiphilic molecules can aggregate to form micelles. While the literature is rich with studies on the properties of micelles, nearly all such studies are concerned with amphiphilic molecules of ordinary molecular size in aqueous systems. Much less attention has been given to polymeric amphiphiles, but regardless of molecular size, efforts to control micelle properties have focused on the chemical identities of the amphiphile's building blocks (e.g., carboxyl, cyano, chloro, alkyl, cycloalkyl, etc.). However, recent studies [1] have shown that micelle properties formed by random copolymers in solution can be controlled not by changing the identity of the building blocks but by adjusting the copolymer's architecture, for example, by the geometric arrangement of the two (or more) building blocks that form the copolymer. Subtle variations in copolymer architecture can have a large effect on micelle formation because such subtle variations can produce a crucial change in conformational entropy, for example, the entropy of the assembled structure (micelle) relative to that of the unassembled (free) copolymers in solution.

To fix ideas, consider a comb polymer where the backbone is polybutadiene (PB) and the branches are polystyrene (PS), dissolved in toluene. For a random graft copolymer (RGC), important variables are $N$, the number of segments in the backbone; $M$, the number of segments in each branch; and $P$, the number of branches per molecule. All branches have the same $M$, and all copolymer molecules have the same $N, M$, and $P$.

Studies with homopolymers in toluene have shown that the Flory parameter $\chi$ for polystyrene is slightly larger than that for polyisobutylene, indicating that the affinity of toluene for the copolymer's backbone is slightly higher than that for the copolymer's branches. This small difference in affinity, however, is sufficient for micellization of the copolymer such that the PS branches form the micelle's core while the PB backbone forms the micelle's periphery called the corona, as shown schematically at the top of Fig. 1.

Using a field theory (too complicated for summary here), keeping $N$ and $M$ constant while varying $P$, Qi and Chakraborty [2] calculated the volume fraction of RGC in toluene where the solution becomes unstable (i.e., where a phase transition occurs); this volume fraction is interpreted as close to the critical micelle concentration (i.e., the concentration of copolymer where micelles are formed). Figure 2 shows results of this calculation where Flory parameter $\chi$ for PB-toluene is set equal to zero and that for PS-toluene is set equal to either 0.10 or 0.07 . The important feature of Fig. 2 is that the critical volume fraction first decreases and then increases with $P$, the number of branches per molecule. For micelle formation, the optimum $P$ is about 7 .

We have convincing experimental evidence for micelle formation for these random graft copolymers (RGC) from dynamic light-scattering data reported by Balsara [1], shown in Figs. 3 and 4. The top part of Fig. 3 shows the time autocorrelation function $\mathrm{g}(\tau)$ for a dilute solution of the RGC polybutadiene with 10 polystyrene branches. An autocorrelation function in excess of unity indicates an 
entropy for PB loops

entropy for packing

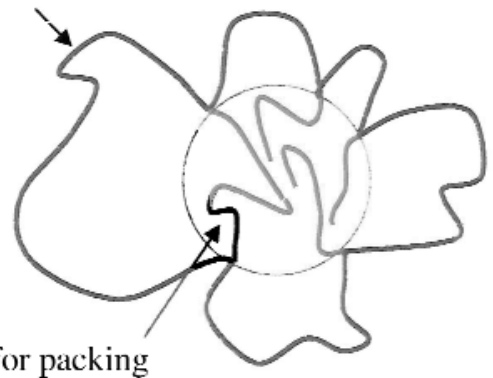

PS core

For small number of branches (P)

- loops are long $(\sim \mathrm{N} / \mathrm{P})$; core is small.

- entropy for packing core dominates.

- entropy penalty decreases as P increases.

For large number of branches (P)

- loops are short ( N/P); core is large.

- loop entropy dominates.

- entropy penalty increases as $\mathrm{P}$ increases.

Fig. 1 Physical reason for optimum.

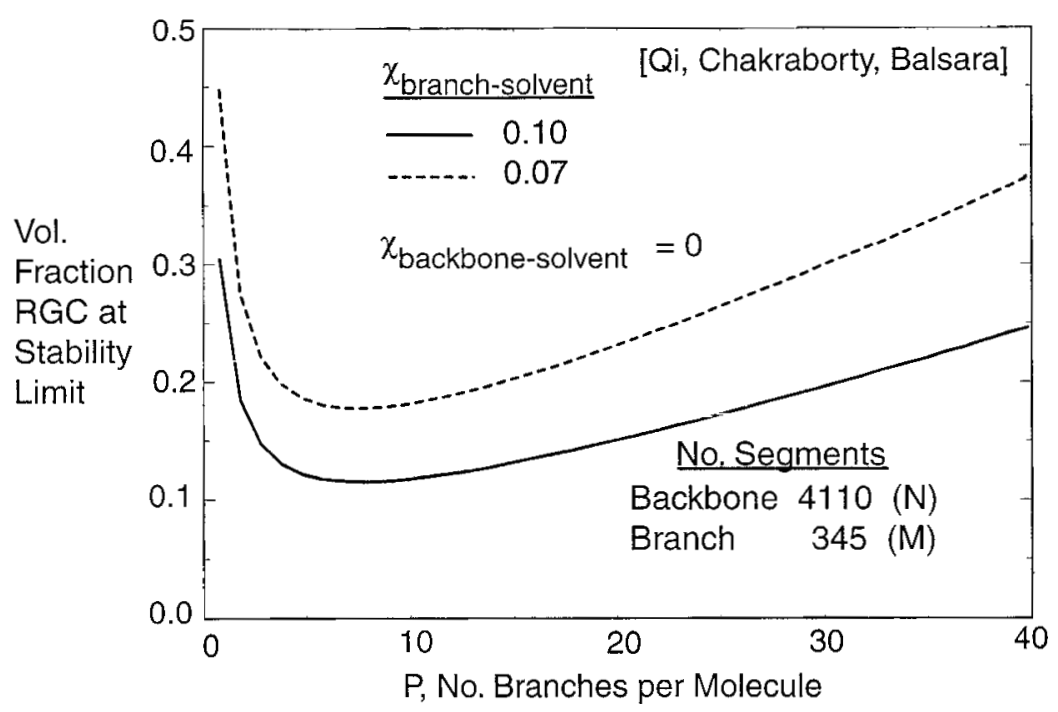

Fig. 2 Phase behavior (spinodal) for RGC solutions.

(C) 2000 IUPAC, Pure and Applied Chemistry 72, 1819-1834 

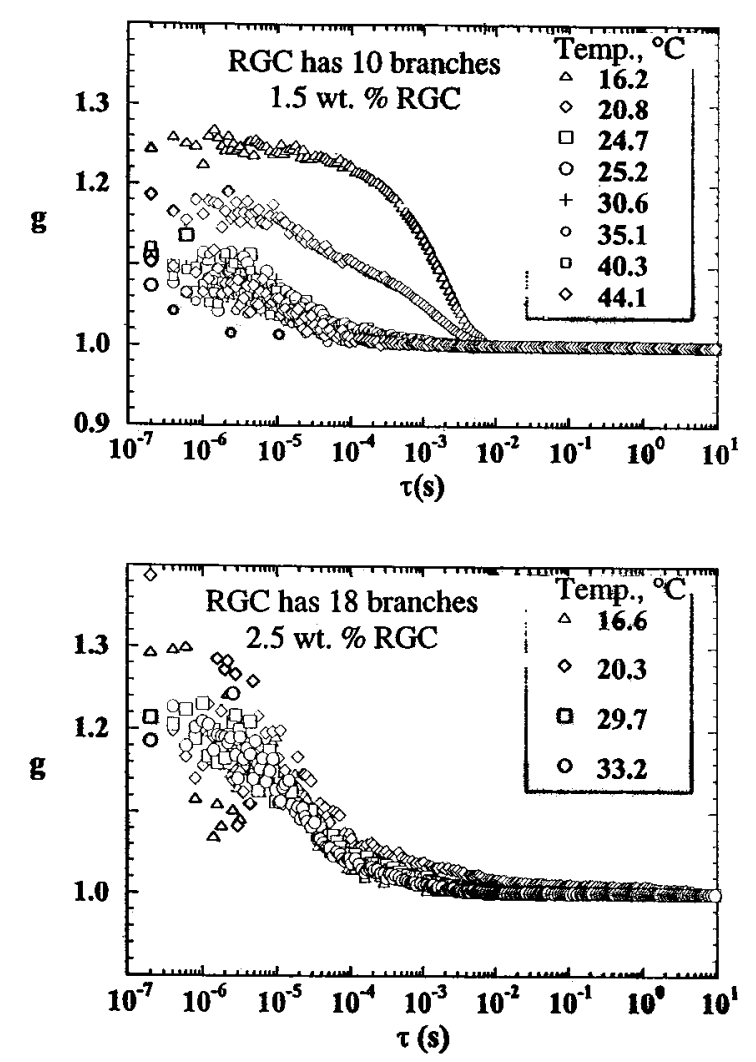

$\mathrm{g}=$ time autocorrelation function $\tau=$ time interval

Fig. 3 Autocorrelation function from dynamic light scattering.

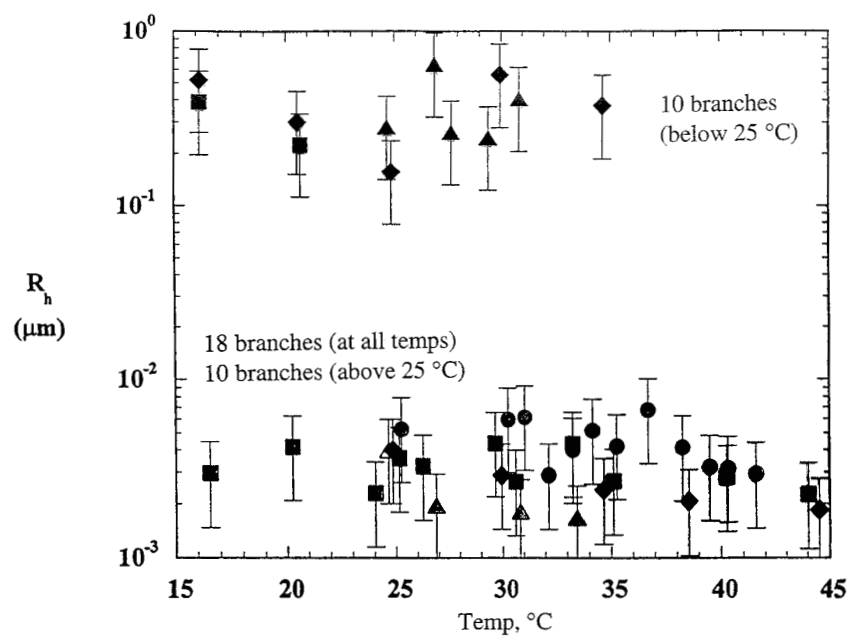

Fig. 4 Hydraulic radius from dynamic light-scattering data. 
assembled structure. In the temperature range 16 to $25^{\circ} \mathrm{C}, \mathrm{g}(\tau)$ remains well in excess of unity for about $10^{-2}$ seconds; at higher temperatures, $\mathrm{g}(\tau)$ decreases toward unity at times well below $10^{-2}$ seconds.

By contrast, the lower part of Fig. 3 shows qualitatively different results for a RGC with 18 branches per molecule at a somewhat higher concentration in toluene. These data show that the plateau for $\mathrm{g}(\tau)$ remains constant, regardless of temperature, for only about $10^{-4}$ seconds, two orders of magnitude lower than that for copolymers with $P=10$.

From scattering theory, and from the Stokes-Einstein equation relating the diffusion coefficient to the hydraulic radius, these light-scattering data give the hydraulic radius of the diffusing species in toluene. Figure 4 shows results indicating that 10-branch copolymers form micelles below $25^{\circ} \mathrm{C}$ while 18 -branch copolymers do not. In these experiments, $M$ and $N$ were held constant $(N=2000 ; M=300)$ while $P$ was either 10 or 18.

We can at least approximately understand why there is an optimum value for $P$ as summarized in Fig. 1. When a micellar assembly is formed from RGCs, there is a decrease in entropy (called the entropy penalty) that must be overcome by a simultaneous decrease in enthalpy. For the micellization process to proceed, $\Delta \mathrm{G}$ (micellization) must be negative:

$$
\Delta \mathrm{G}=\Delta \mathrm{H}-\mathrm{T} \Delta \mathrm{S}<0
$$

In a solvent that prefers backbone over branches, branches aggregate. When branches aggregate, the backbone forms a shroud (corona) around the assembled branches, as shown in Fig. 1. If the loops are long, micellization has little effect on the entropy of the loops; the entropy of the branches is dominant. As $P$ rises, it is increasingly difficult for the branches to form a densely packed structure because they are restrained by the backbone. Hence, the entropy penalty decreases with rising $P$.

However, as $P$ rises more, the entropy of the corona loops becomes increasingly important because the freedom of loop segments to move declines as the loop length decreases. For large $P$, therefore, the loop entropy becomes dominant and the entropy penalty increases with rising $P$.

These initial results suggest further studies. For possible medical applications, a more interesting $\mathrm{RGC}$ is one where the backbone is hydrophobic and the branches are hydrophilic. Such RGCs now under investigation by Hagan, Chakraborty, and Balsara (University of California, Berkeley), may form cylindrical micelles with top and side views as shown in Fig. 5. These cylindrical micelles could, perhaps, be used for a drug-delivery device by forming nanotubes that could transport a hydrophilic solute (i.e., a drug) through a membrane where hydrophilic (light gray) layers alternate with hydrophobic (white) layers as shown in Fig. 6.

On the left side of Fig. 6, the RGC, soluble in the hydrophobic layer, is not assembled. When the concentration of the RGC reaches the critical micelle concentration, a micelle is formed with (perhaps) cylindrical shape. When activated by some switch (Fig. 7), this micelle could form a pore permitting a hydrophilic drug to pass from one hydrophilic layer to another.

This potential device is still in its infancy; for its development, much experimental work lies ahead. However, the experimental program for design can be much aided by molecular-thermodynamic calculations. Such calculations provide guidance to indicate the most promising RGC for achieving the desired micelle properties.

It is not necessary here to present details of such calculations; however, the general idea is briefly outlined in Fig. 8 where the Helmholtz energy $F$ of the RGC in the hydrophobic phase is given as the sum of three contributions. The most important of these is $F^{(1)}$, the Helmholtz energy of the micelle relative to the indicated reference state. Some important factors contributing to $F^{(1)}$ are shown in Fig. 9.

The Helmholtz energy of the system is minimized with respect to the most important variables identified in Fig. 8. At the minimum, the values of these variables are then determined.

The calculations indicated here are far from trivial and while much is known, much further theoretical work is required to obtain reliable results. While these calculations by themselves are not sufficient for supplying the final answers required for design, they facilitate interpolation and extrapolation 

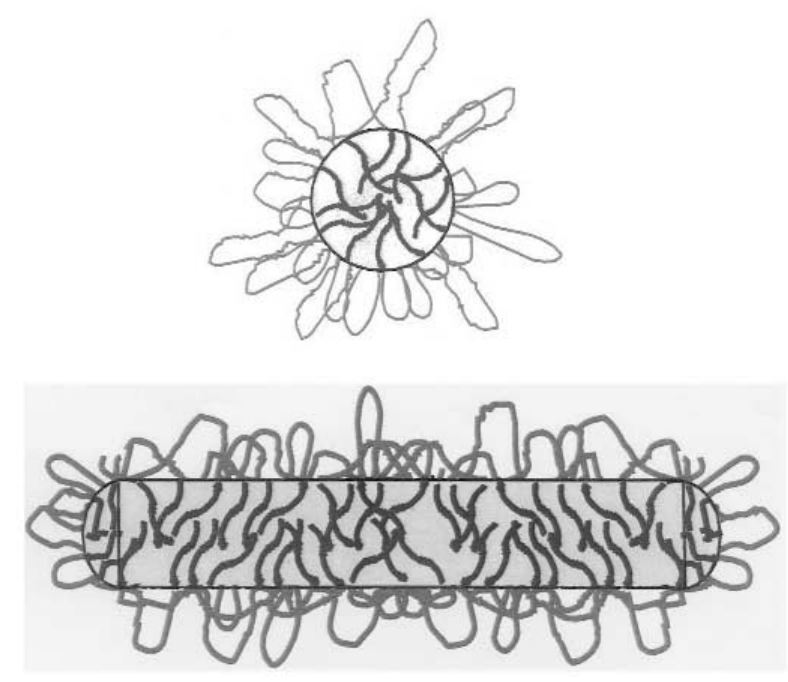

\section{Hydrophobic (light gray) Hydrophilic (dark gray) \\ Polymer Backbone \\ Micelle Corona \\ Polymer Branches \\ Micelle Core}

Fig. 5 Formation of micelles with random graft copolymers, as suggested by Hagan, Chakraborty, and Balsara.

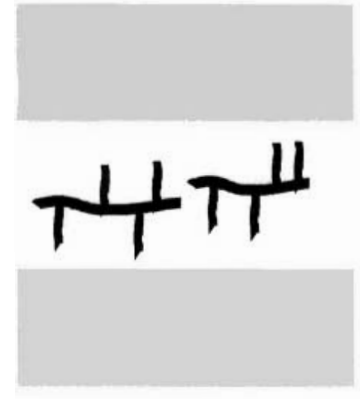

I

Bilayer with RGC randomly dispersed in hydrophobic layer
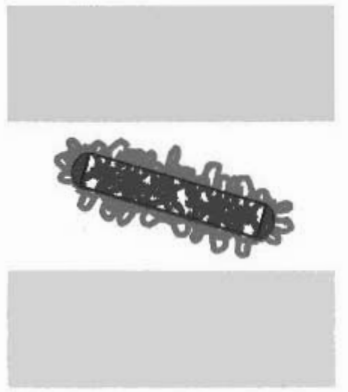

(a)

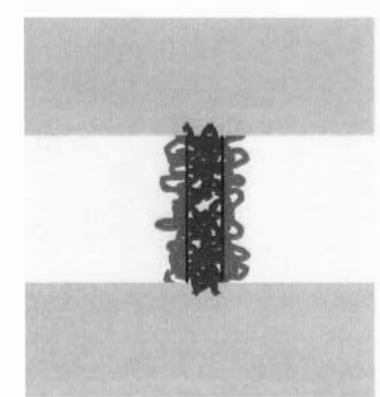

II

RGC self-assemble to form cylindrical micelles in hydrophobic layer
(a) Random orientation
(b) Vertically aligned

Fig. 6 Synthetic channel (pore) for transport through a bilayer. 


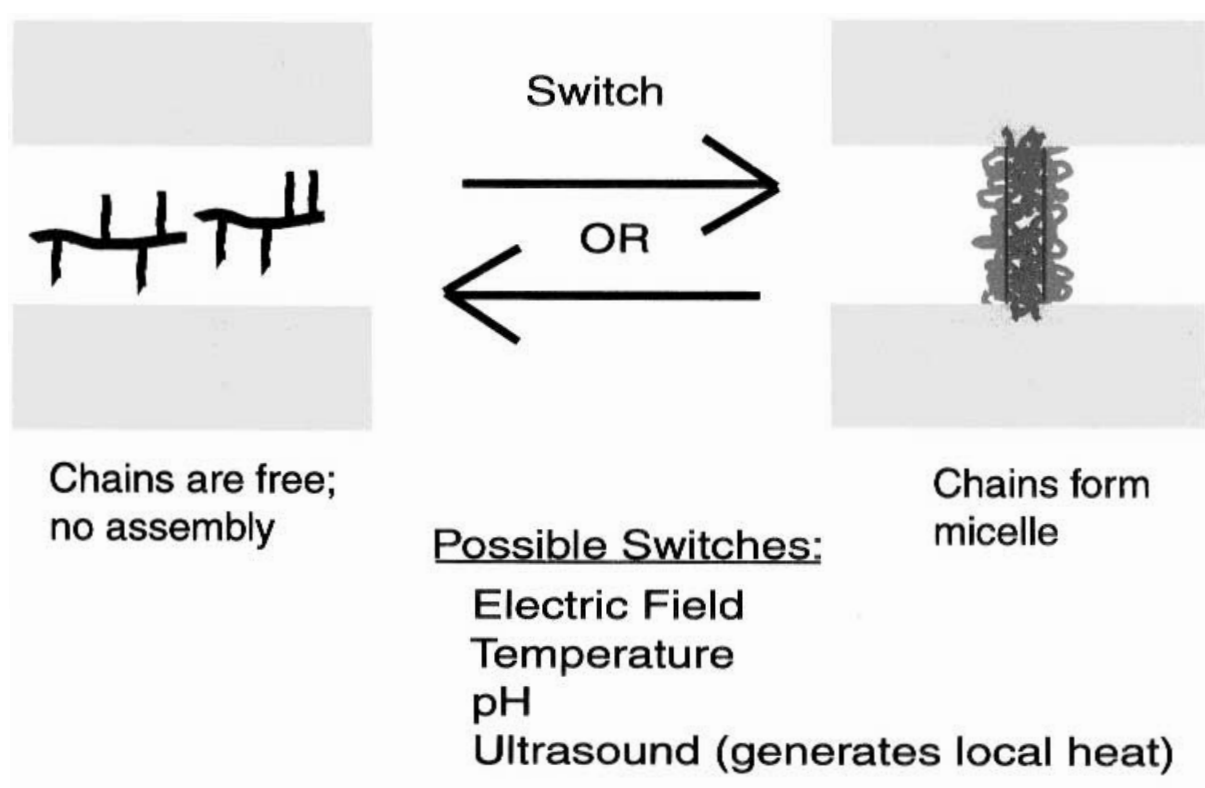

Fig. 7 Synthetic channel (pore) for transport of a hydrophilic solute (drug) through a bilayer.

Reference state is pure solvent and pure copolymer melt

(1) $\quad F\left(\right.$ system) $=F_{\text {micelle }}^{(1)}+\mathbf{F}_{\substack{\text { unassembled } \\ \text { chains }+ \text { solvent }}}^{(2)}+\mathbf{F}_{\text {mixing micelles with }}^{(3)}$

$$
\begin{aligned}
& \mathbf{F}^{(2)}=\text { Flory-Huggins equation } \\
& \mathbf{F}^{(3)}=\text { Ideal Mixing } \\
& \mathbf{F}^{(1)}=\mathscr{F}\left(\mathbf{N}_{c}, \eta, \xi, \mathbf{L}\right) \\
& \mathbf{N}_{\mathbf{c}}=\text { Number of chains per micelle } \\
& \eta=\text { Volume fraction of copolymer monomers in corona } \\
& \xi=\text { fraction of copolymer that has aggregated } \\
& \mathbf{L}=\text { length of micelle cylinder }
\end{aligned}
$$

Minimize Eq. (1) with respect to $N_{c}, \eta, \xi$, and $L$.

Fig. 8 Helmholtz energy of a micellar system containing copolymer and solvent. 
Contributions to $\mathbf{F}^{(1)}$ from

a. Corona-core interface

b. Deformation (Loss of configurational entropy) for chains that form the core and the corona, including loop entropy

c. Mixing of corona and solvent

For example, for part a:

$\mathbf{F}_{(a)}^{(1)}=($ surface area of micelle core) $\gamma$

$\gamma=\left(\mathrm{kT} / \ell^{2}\right)(\chi / 6)^{1 / 2}$

$\chi=$ Flory parameter for backbone-branch interaction (assuming that $\chi_{\text {backbone-sotvent }}=0$ )

$\ell=$ Kuhn length of copolymer segment

$k=$ Boltzmann constant

The main reason for an optimum number of branches follows from contributions from loop entropy to $F^{(1)}$.

Fig. 9 Key function $\mathrm{F}^{(1)}$ for minimizing the Helmholtz energy.
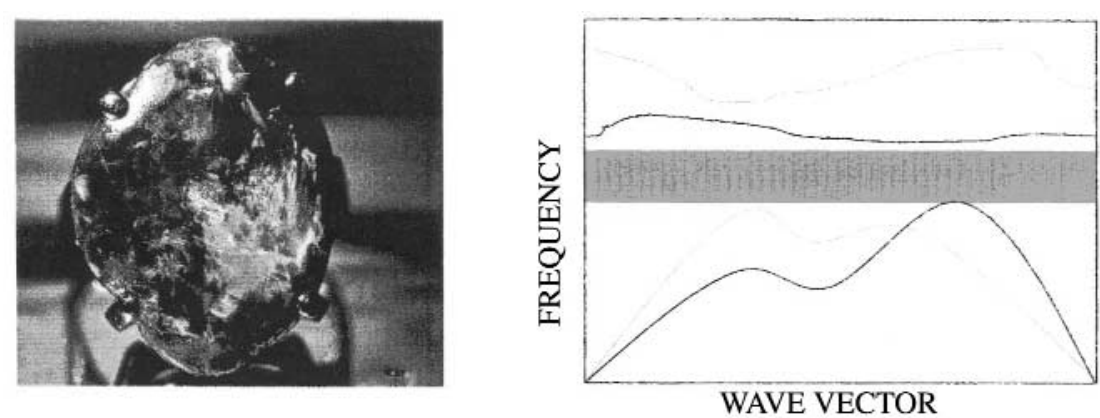

- control of emission in semiconductors

- microcavity light-emitting diodes (MCLEDs)

- lasers for telecommunication applications

- optical circuits

Fig. 10 Programmed assembly toward colloidal crystals. Photonic-band-gap materials. 
of experimental results, and they provide first estimates of where to find the optimum conditions for a particular application.

It was Clausius, one of the founders of thermodynamics, who said "Nothing is more practical than a good theory".

This first example considered chain molecules of complex size and shape; the interesting phenomena arise from flexibility. The second example considers a suspension of spherical particles where flexibility plays no role at all; instead, the interesting phenomena arise because the suspension is placed into a severely confined space where the freedom of the particles depends on the extent of confinement.

\section{COLLOID CRYSTALLIZATION IN A CONFINED SPACE}

Colloidal crystals are of potential interest as new catalysts or catalyst supports and as membranes for separations. However, perhaps the most important potential use for these crystals is as components in optical circuits and as light-emitting diodes.

The optical properties of colloidal crystals depend not only on the chemical nature of the colloidal particles but also on their geometric arrangement in space (i.e., on crystal structure). To illustrate, the left side of Fig. 10 shows a black-and-white picture of a piece of jewelry, an opal mounted on a ring. Opal is a naturally occurring colloidal crystal; the opal's variety of colors follows from an assembly of slightly different crystal structures.

For application in optical circuits, electrical engineers seek a crystal that is a photonic-gap-band material as indicated on the right side of Fig. 10. Knowing the dielectric constant of the particles that comprise the crystal, and knowing the geometry (structure) of the crystal, a set of solutions can be obtained to the Maxwell equations for electromagnetic radiation; these solutions give light frequency as a function of wave vector. Desired is a crystal where all possible solutions to Maxwell's equations do not enter a region of frequency-wave number space, the so-called band gap shown in Fig. 10. While the search for a suitable band-gap crystal is necessarily experimental, that search can be much aided by statistical thermodynamics.

Preparation of a photonic-band-gap colloidal crystal is generally not possible under ordinary conditions. One possible way to achieve the desired structure is to place the colloidal solution between two solid walls; the two walls are brought very close to each other such that the separation $H$ between the plates is of the order of $\sigma$, the diameter of the colloidal particles (i.e., the ratio $H / \sigma$ should be in the range 1-10). Under such close confinement, it is possible to achieve crystal structures that cannot be attained under normal conditions. For a typical colloidal particle, $\sigma=100 \mathrm{~nm}$.

Statistical thermodynamics can be used to generate a phase diagram for colloids under a closeconfinement restraint. This phase diagram gives the boundaries of the fluid phase and of several possible crystal phases.

Consider a colloid between two horizontal solid walls. Omitting ideal-gas contributions, the Helmholtz energy $F$ of the fluid phase is given by

$$
F=A \int_{0}^{\mathrm{H}} \rho(z) f(\bar{\rho}) \mathrm{d} z
$$

where $A$ is the area of the wall and $z$ is the coordinate perpendicular to the wall; $\rho(z)$ is the density profile of the colloid. Lower case $f$ denotes specific Helmholtz energy (i.e., Helmholtz energy per particle). Because the walls exercise a strong influence on the free volume available to the colloid particles, the colloid's density is not everywhere the same; indeed, it is precisely because of the wall effect that it is possible to achieve crystal structures that are not attained at normal conditions.

In eq. 1, specific Helmholtz energy $f$ is that of a homogeneous fluid (no wall effect) at some suitably weighted average density p. (A variety of proposals, not discussed here, have been made for eval- 
uating $p$.) The specific Helmholtz energy $f(p)$ is determined from properties of the bulk (not confined) fluid.

The density profile is determined by an equilibrium condition; it is that profile which satisfies

$$
\frac{\delta F}{\delta \rho(z)}=0
$$

where the left side of eq. 2 is a functional derivative. After the density profile has been established, the chemical potential of the fluid $\mu$ is found from ordinary (not functional) differentiation:

$$
\mu=\left(\frac{\delta F}{\delta N}\right)_{\mathrm{T}, V}
$$

where $N$ is the number of particles and $V$ is the volume of the confined colloid.

For a crystal phase, the chemical potential (omitting ideal-gas contributions) is found from cell theory where a particle is confined to a cell formed by nearest-neighbor particles. In cell theory, chemical potential $\mu$ is determined by free volume $V_{f}$ per particle:

$$
\mu=-\mathrm{kT} \ell \mathrm{n} \frac{V_{\mathrm{f}}}{\sigma^{3}}
$$

To calculate free volume, we assume a particular crystal structure, and we use a pertinent twobody potential for the colloidal particles; for most purposes (if the particles are not charged), a hardsphere potential is satisfactory.

For any given fluid height $H$, number of particles $N$ and volume $V$, the stable phase, liquid or (assumed) crystal, is the one with the lowest chemical potential $\mu$.

Figure 11 shows five crystal structures. The lower right side, shows a phase diagram calculated with statistical thermodynamics; the vertical axis gives the normalized distance between plates, and the horizontal axis gives the reduced density. The hatched areas show simultaneous existence of two phases. The forbidden region indicates that region where (because of the very high density) neither fluid nor crystal can exist.

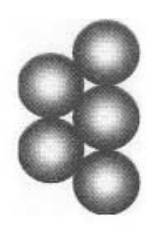

$1 \Delta$

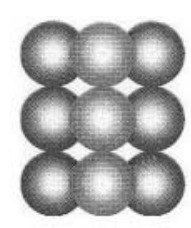

buckling (b)

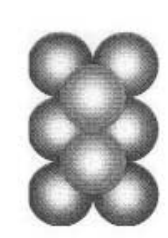

$2 \Phi$

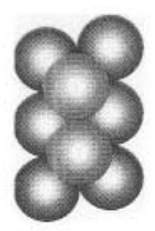

rhombic (r)

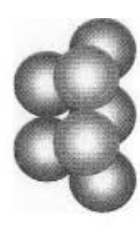

$2 \Delta$
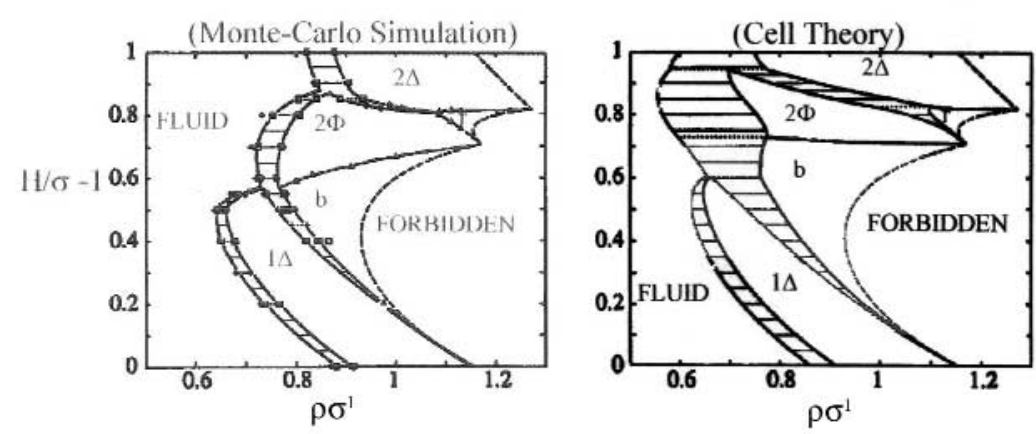

H: distance between plates; $\sigma$ : particle diameter; $\rho$ : density

Fig. 11 Phase diagram for hard spheres confined between two walls. In (b), the three molecules along a vertical line in the center are in a plane slightly above that of the other molecules. 
The lower left side shows the same phase diagram obtained from molecular simulations. The two phase diagrams are in essential agreement although there are some differences, especially in the sizes of the two-phase regions. These diagrams are from Schmidt and Löwen [3].

Figure 12 shows some experimental results for a colloid, obtained by Murray et al. [4]. The horizontal axis is the reduced fluid height; $\sigma$ is the particle diameter. The vertical axis is (essentially) the cube root of the reduced volume. These data for monodisperse polystrene spheres in water were obtained by direct observation with a powerful microscope. In this diagram the hatched areas are not two-phase regions; they are used only as a guide to the eye. However, the close-hatched region between structures $1 \triangle$ and $2 \square$ denotes a two-phase region called "hopping"; $\triangle$ and $\square$ indicate type of structure, while the preceding number indicates the number of colloidal-particle layers in the crystal. Statistical-thermodynamic calculations can reproduce this experimental phase diagram with good agreement.

For charged particles, calculations are more realistic when a Yukawa potential is added as a perturbation to the hard-sphere potential as indicated in Fig. 13. In the Yukawa potential, interaction length $\mathrm{\kappa}^{-1}$ is similar to the Debye length in the familiar Derjaguin-Landau-Verwey-Overbeek (DLVO) theory for colloids. A calculated phase diagram, shown on the left in Fig. 13, gives phase boundaries for the fluid phase, for two crystal phases, and for a glassy phase.

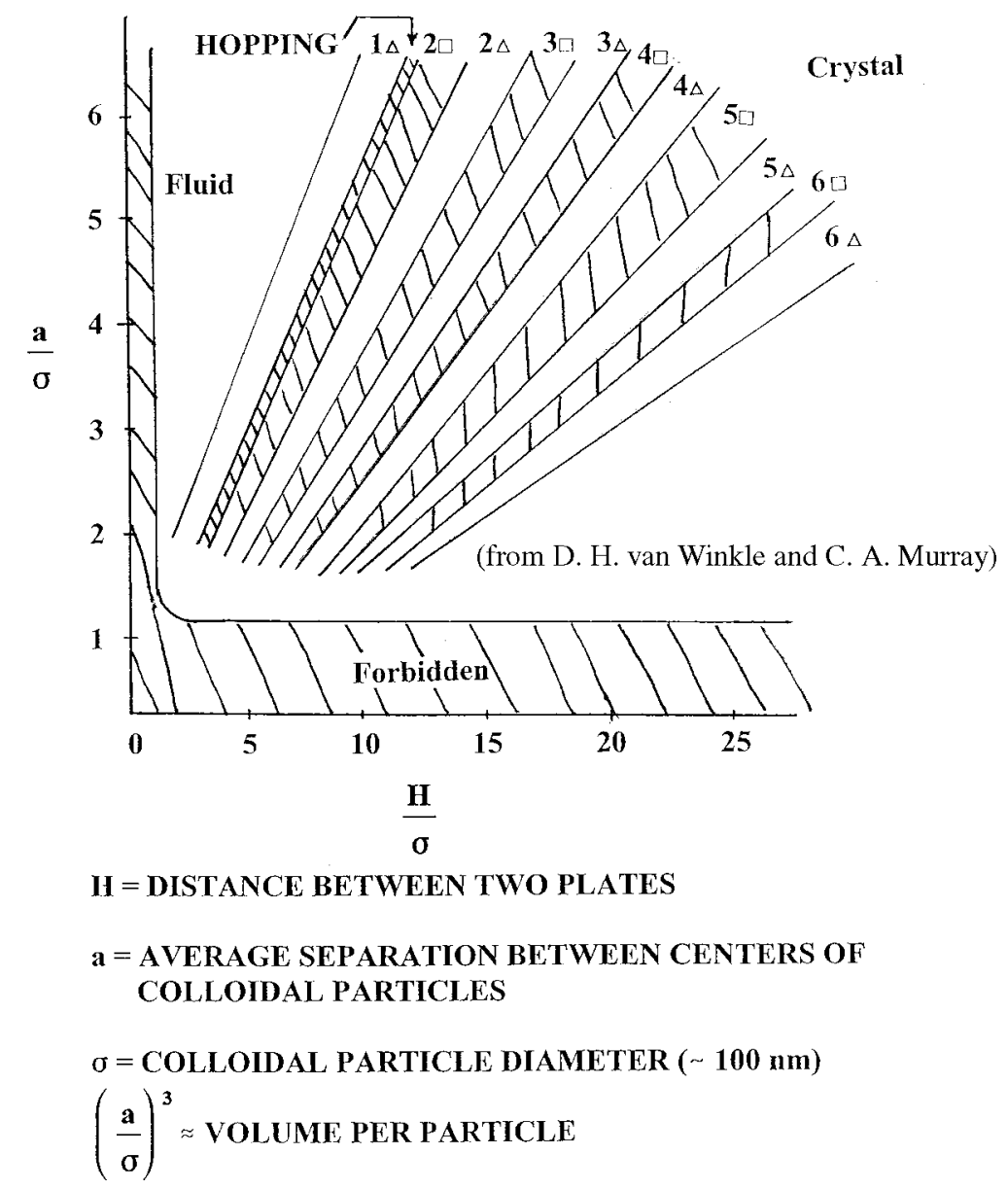

Fig. 12 Observed phase diagram for a confined colloidal solution. 


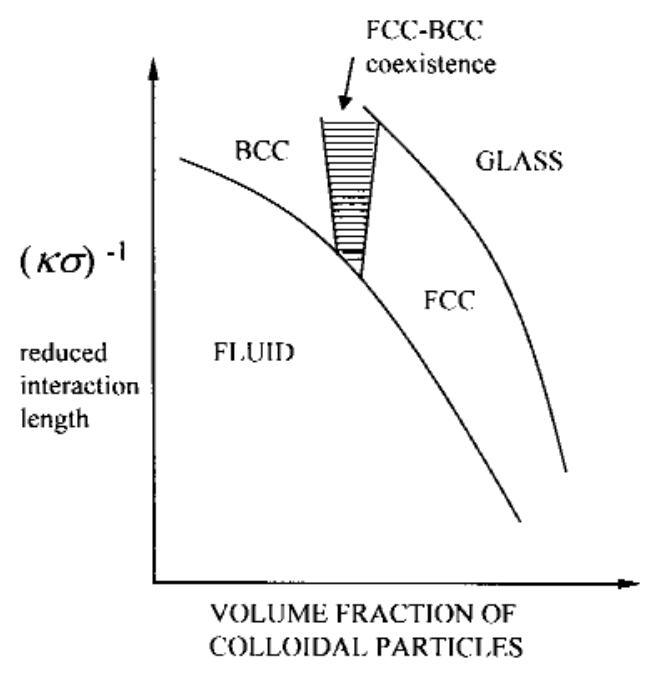

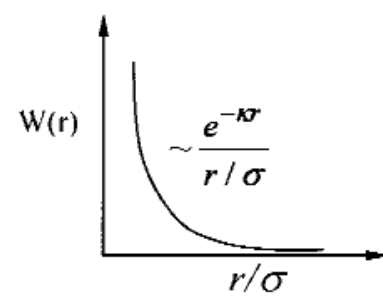

Yukawa Potential

Fig. 13 Phase diagram for a charged colloid calculated with a Yukawa potential. BCC=body-centered cubic. $\mathrm{FCC}=$ face-centered cubic.
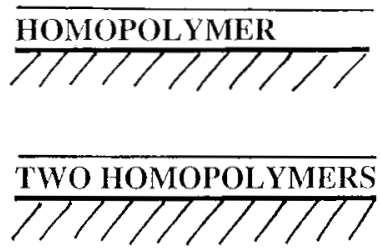

$\overline{\text { RANDOM COPOLYMER }}$
NO PATTERN

MACRO DEMIXING. NO PATTERN

\section{NO PATTERN}

TWO HOMOPOLYMERS A AND B WITH SMALL CONCENTRATION OF COMPATILIZING BLOCK COPOLYMER A $\cdots$ B

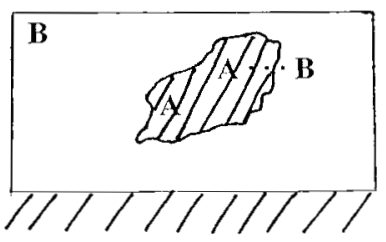

SHADED AREA IS A MICRODOMAIN OF A IN CONTINUOUS B STABILIZED BY BLOCK COPOLYYMER A $\cdots$ B

REGRETTABLY, FOR BULK FILMS, THIS DOES NOT WORK BECAUSE BLOCK COPOLYMERS A - . B FORM MICELLES; THEY DO NOT STAY AT THE A-B INTERFACE.

Fig. 14 Possible pattern formation in a polymer film.

In the search for a photonic-band-gap material, statistical thermodynamics is useful for determining what crystal structures may be obtained when crystallization occurs under a geometric restraint. 
Using the Maxwell equations, it is then possible to determine if that structure has the desired band-gap property.

As in the previous example, statistical thermodynamics cannot replace experimental effort but it can significantly reduce that effort by providing guidance to the experimentalist.

The first example serves to illustrate the effect of molecular flexibility on micellization of a graft comb copolymer. The second example serves to illustrate the effect of confinement on the phase behavior of a suspension of spherical particles where flexibility plays no role. In the final third example, we consider a combination: suppression of micellization of a flexible block copolymer in a confined thin film supported by a wall on one side and a vapor-liquid interface on the other.

\section{PROPERTIES OF A THIN FILM OF BLOCK COPOLYMER}

For possible use in lithography, we want a thin polymer film that can give a pattern on a solid surface. A film of a homopolymer or a random copolymer will not give a pattern. A mixture of two polymers with microdomains could give a pattern but because, with rare exceptions, two polymers are almost always immiscible, a stable mixture cannot be made without addition of a small amount of a third component (compatibilizer). For polymer A and polymer B, a suitable third component is a two-block copolymer A $\cdots$ B $\cdots$. Only a very small amount of block copolymer would be needed to stabilize the A-B mixture because in continuous phase $\mathrm{B}$, a small domain of $\mathrm{A}$ is stabilized by the block copolymer at the domain interface where the A part of the block copolymer is soluble in domain A and the B part of the block copolymer is soluble in continuous phase B. Regrettably, this reasonable idea is not practical because, instead of remaining at the domain interface, the block-copolymer molecules prefer to form a micelle. When that occurs, the mixture of polymers A and B is unstable and splits into one A-rich phase and one B-rich phase. These ideas are illustrated in Fig. 14.

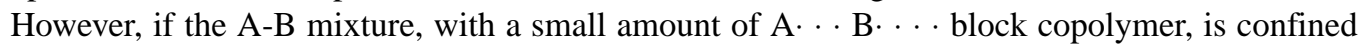
to a thin film, micellization can be suppressed as indicated in Fig. 15. The left side (I) shows a polymer film where the film thickness $h$ is sufficiently large to allow micellization of the block copolymer; phases $\mathrm{A}$ and $\mathrm{B}$ are not miscible. The right side (II) shows a thin film with a smaller thickness $h$ such that

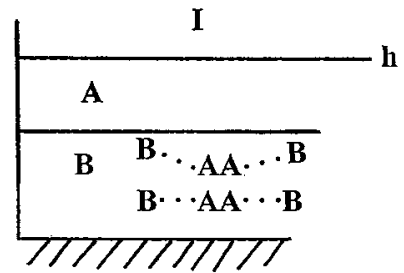

THICK FILM. MICELLES PREVENT A $\cdots$ B FROM STABILIZING DOMAINS OF A IN B.

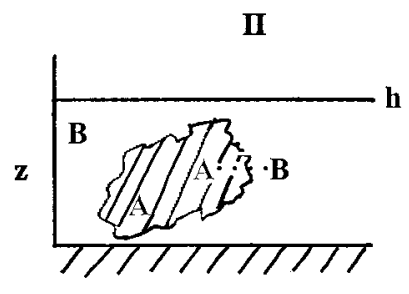

THIN FILM. PRESENCE OF WALL PREVENTS MICELLE FORMATION.

\section{WE CALCULATE FREE ENERGY F(h) TWICE, ONCE ASSUMING STRUCTURE I AND ONCE ASSUMING STRUCTURE II.}

$\begin{array}{lll}\text { FOR } h>H & F_{I}<F_{I I} & \text { I IS STABLE } \\ \text { FOR } h<H & F_{1}>F_{I I} & \text { II IS STABLE }\end{array}$

Fig. 15 Suppression of micellization due to a wall. 
micellization cannot occur. The block copolymer stabilizes the (shaded) domain A in continuous phase B. The task of statistical mechanics is to determine the critical film thickness $H$ such that for $h>H, F_{\mathrm{I}}$ $<F_{\text {II }}$ (where $F$ is Helmholtz energy) and for $h<H, F_{\mathrm{I}}>F_{\text {II. }}$ To determine that critical film thickness, we require $F(h)$. Nature gives us the state with the lower Helmholtz energy. For a given $h$, does State I or State II have the lower Helmholtz energy?

It is not necessary here to present in detail how the calculation is made; a summary is sufficient.

Per unit area of the supporting wall, the Helmholtz energy of the mixture, relative to those of the pure components, is given by

$$
F(h)=-\mathrm{kT} \ell \mathrm{n} \Omega(h)+\chi \int_{0}^{\mathrm{h}} \Phi_{\mathrm{A}}(z)<\Phi_{\mathrm{B}}(z)>\mathrm{d} z
$$

where $z$ is the coordinate perpendicular to the wall, $\chi$ is the Flory interaction parameter for the A-B pair and $\Phi$ denotes volume fraction. Because the wall influences the properties of the mixture, volume fractions $\Phi_{\mathrm{A}}$ and $\Phi_{\mathrm{B}}$ depend on $z$; that dependence is called the volume-fraction profile. The brackets in eq. 5 indicate a local average; at position $z$, the volume fraction of $A$ is $\Phi_{A}(z)$. The average volume fraction of $\mathrm{B}$ for a differential volume of height de in the immediate neighborhood of A divided by $\mathrm{dz}$ is given by $\left\langle\Phi_{\mathrm{B}}(z)>\right.$.

The most important quantity, $\Omega(h)$, is the configurational factor that describes the number of possible configurations of chains A, B and A $\cdots$ B $\cdots$ for a film of height $h$ with density profile $\Phi_{\mathrm{A}}(z)$. A lattice theory can be used to calculate this combinatorial factor for a given volume-fraction profile and for a fixed $h$. This is a difficult (but possible) calculation because the volume-fraction profile and the combinatorial factor must be determined simultaneously using a self-consistent lattice theory where each lattice point is occupied by one polymer segment. Here, "self-consistent" means that the correct combinatorial factor and volume-fraction profile are those that correspond to a global minimum of the Helmholtz energy.

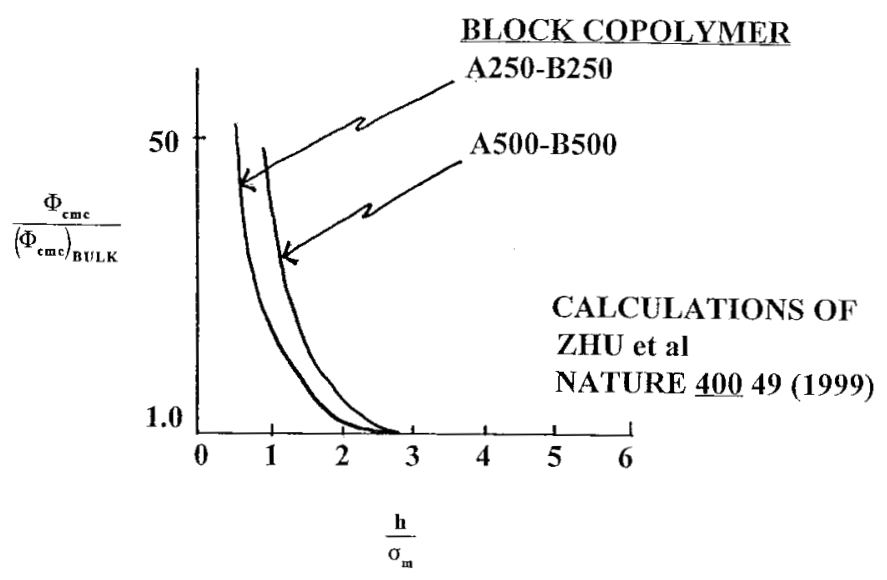

$$
\begin{aligned}
\sigma_{\mathrm{m}}= & \text { MICELLE DIAMETER IN BULK PHASE } \\
\Phi_{\mathrm{cmc}}= & \text { CRITICAL MICELLE CONCENTRATION } \\
& \text { OF BLOCK COPOLYER A } \cdots \text { B }
\end{aligned}
$$

h = FILM THICKNESS

Fig. 16 Critical micelle concentration depends on film thickness. 


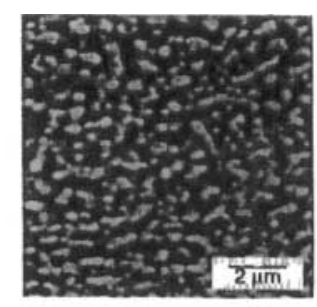

$\mathbf{h}=1000 \AA>H$
FROM S. ZHU

et al, NATURE

40049 (1999).

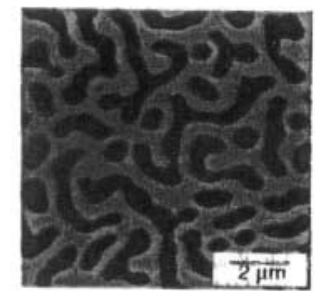

$\mathbf{h}=\mathbf{5 0 0} \AA<\mathbf{H}$

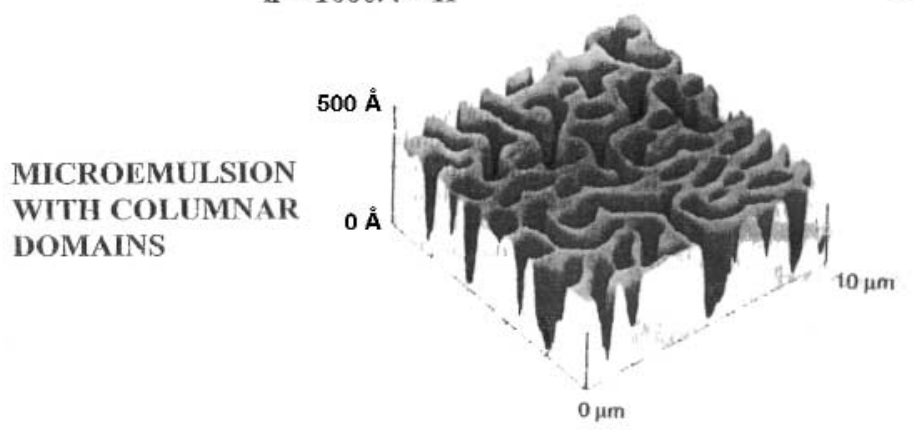

IN THESE PICTURES, AFTER EQUILIBRIUM, PS WAS REMOVED WITH CYCLOHEXANE.

Fig. 17 Microscopic images for mixtures of polystyrene and polymethylmethacrylate with a small amount of diblock copolymer.

Figure 16 shows illustrative calculations performed by Zhu et al. [5]. The horizontal axis is the dimensionless film height. The vertical axis gives the critical micelle concentration in the film normalized by that in the bulk phase. Two calculations are shown; one is for a symmetric block copolymer with 250 segments of A and 250 segments of B; the other is for an asymmetric block copolymer A250-B500.

For dimensionless film heights below about 2.5 , the ratio on the vertical axis is larger than unity. For these thin films, the permissible concentration of block copolymer may be sufficiently large to stabilize domains of $\mathrm{A}$ in a continuous $\mathrm{B}$ phase.

Finally, Fig. 17 shows some experimental results from Zhu [4] for mixtures of PS and polymethyl methacrylate (PMMA). Shown on the left is a scanning-force microscopy image for a film height above the critical height $H$ while that on the right is for a film height below $H$. The picture on the left indicates undesired separated phases of A and B while that on the right indicates desired microdomains of $\mathrm{A}$ in continuous B. These microdomains may be useful for lithography. The bottom picture gives a three-dimensional image corresponding to the picture on the right.

As briefly outlined here, theoretical calculation of critical film thickness $H$ can provide a useful guide for the experimentalist.

\section{CONCLUSION}

The examples selected here were chosen to give the flavor of a major frontier of modern chemical thermodynamics. While creation and understanding of soft materials depend primarily on experimental science, statistical mechanics is now able to provide guidance toward interpreting experimental results and toward reducing experimental effort. While molecular-thermodynamic models should never be identified as true reality, they are useful for suggesting what experimental work is most likely to lead to a successful result. At this time, application of hard science to soft materials is still in an early stage but there is good reason to expect that, thanks to the creative efforts of dedicated researchers, such application is likely to grow dramatically in the near future. 


\section{ACKNOWLEDGMENTS}

For financial support, the author is grateful to the National Science Foundation and to the Director, Chemical Sciences, Office of Basic Energy Sciences, U.S. Department of Energy. For advice and helpful discussions, the author is grateful to his co-worker, J. Z. Wu, to Berkeley colleagues N. Balsara and A. Chakraborty, and to their co-worker M. Hagan.

\section{REFERENCES}

1. S. Qi, A. Chakraborty, H. Wang, A. Lefebvre, N. Balsara, E. Shakhnovich, M. Xenidou, N. Hadjichristidis. Phys. Rev. Lett. 82, 2896 (1999); and A. Lefebvre et al. J. Chem. Phys., submitted for publication.

2. S. Y. Qi and A. Chakraborty. J. Chem. Phys. 112, 1585 and 1598 (2000).

3. M. Schmidt and H. Löwen. Phys. Rev. Lett. 76, 4552 (1996).

4. D. H. van Winkle and C. A. Murray. Phys. Rev. A 34, 562 (1986).

5. S. Zhu, Y. Liu, M. H. Rafailovich, J. Sokolov, D. Gersappe, D. A. Winesett, H. Ade. Nature 400, 49 (1999). 